\title{
LINEARIZATION STABILITY OF THE EINSTEIN EQUATIONS
}

\author{
BY ARTHUR E. FISCHER ${ }^{1}$ AND JERROLD E. MARSDEN ${ }^{1}$
}

Communicated by R. Seeley, January 8, 1973

1. Introduction. An important problem in general relativity is the question of whether or not a solution of the linearized Einstein field equations (relative to a given background solution) actually approximates to first order a curve of exact solutions to the nonlinear equations. Here we announce that under certain geometrical conditions on the background solution the problem can be answered affirmatively; however, in certain exceptional cases the answer may be negative. In the affirmitive case we shall say that the background metric is linearization-stable.

Let ${ }^{(4)} g$ be a Lorentz metric (signature,,,-+++ ) on a 4-manifold $V$. The empty space Einstein field equations of general relativity are that the Ricci tensor of ${ }^{(4)} g$ vanish:

$$
\operatorname{Ric}\left({ }^{(4)} g\right)=0 .
$$

By an infinitesimal deformation we mean a 2-covariant symmetric tensor field ${ }^{(4)} h$ which satisfies the linearized equations:

$$
D \operatorname{Ric}\left({ }^{(4)} g\right) \cdot{ }^{(4)} h=0 \text {, }
$$

where $D \operatorname{Ric}(\cdot)$ is the derivative of the map $\operatorname{Ric}(\cdot)$.

Assume that $V$ has a compact orientable space-like hypersurface $M$ and let $g$ denote the induced Riemannian metric and $k$ the second fundamental form. Our conditions are as follows:

(i) $\operatorname{tr} k$ ( $=$ trace of $k$ ) is constant on $M$.

(ii) If $k=0, g$ is not flat.

(iii) There are no nonzero vector fields $X$ such that $L_{X} g=0$ and $L_{X} k=0 ; L_{X}$ denotes the Lie derivative.

Under these conditions, every solution ${ }^{(4)} h$ of (2) is tangent to a curve ${ }^{(4)} g(\lambda)$ of exact solutions of $(1)$; i.e. ${ }^{(4)} g(0)={ }^{(4)} g$ in a tubular neighborhood of $M$ in $V$, and $d^{(4)} g(\lambda) /\left.d \lambda\right|_{\lambda=0}={ }^{(4)} h$ in this neighborhood.

The case of noncompact $M$ is rather different. Here asymptotic conditions are necessary. For example, $k=0$ and $g$ the usual flat metric on $\boldsymbol{R}^{3}$

AMS (MOS) subject classifications (1970). Primary 83C05; Secondary 58D15, 83C25, $53 \mathrm{C} 25$.

Key words and phrases. General relativity, Einstein equations, linearization, stability, Ricci flat, nonlinear differential equations.

${ }^{1}$ Partially supported by NSF grants GP-39060 and GP-8257. 
is not excluded. Thus the usual Minkowski metric on $\boldsymbol{R}^{4}$ is linearization stable in a tubular neighborhood of the hypersurface $M=R^{3}$. This result was obtained independently using other methods by Y. ChoquetBruhat and S. Deser [5]. The treatment of the general noncompact case is in spirit similar, although there are certain technical difficulties associated with elliptic operators which enter the problem. Details of this and of the present work will appear elsewhere [11].

2. Notation and summary of the method. Let $M$ be a compact oriented 3-manifold without boundary. Let $\mathscr{M}$ be the space of $C^{\infty}$ Riemannian metrics on $M, S_{2}$ the space of 2-covariant symmetric tensor fields on $M$, $S^{2}$ the 2-contravariant symmetric tensor fields, $C^{\infty}$ the smooth scalar functions on $M$, and $\mathscr{X}$ the smooth vector fields on $M$.

In the technical proofs it is necessary to use the Sobolev $H^{s}$ topology on these spaces and to pass to $C^{\infty}$ by a regularity argument (as in [6]), but here we shall work directly in $C^{\infty}$ for simplicity.

As is well known [1], [8], every space-like hypersurface in a Ricci flat Lorentz manifold satisfies the constraint equations:

$$
\begin{aligned}
\mathscr{H}(g, \pi) & =\left(\frac{1}{2}(\operatorname{tr} \pi)^{2}-\pi \cdot \pi\right)+R(g)=0, \\
\delta(g, \pi) & =-\pi_{\mid j}^{i j}=0 .
\end{aligned}
$$

Here $g$ is the metric induced on the hypersurface, $R$ is the scalar curvature of $g$, and $\pi=((\operatorname{tr} k) g-k)^{-1} \in S^{2}$ where $k$ is the second fundamental form, and ${ }^{-1}$ denotes the contravariant form of a covariant tensor. Also, $\pi \cdot \pi=\pi^{i j} \pi_{i j} \in C^{\infty}$. Note that $\pi$ is often taken to be a tensor density as in [1], [3] and [4], but here it is a tensor.

Conversely, every solution $(g, \pi)$ of (3) gives rise to a Ricci flat spacetime in a neighborhood of the hypersurface; see [9] and references therein.

The solutions of the constraint equations may be regarded as a certain subset $\mathscr{C}$ of the tangent bundle of $\mathscr{M}, T \mathscr{M} \approx \mathscr{M} \times S_{2} \approx \mathscr{M} \times S^{2}$. Our method is to show that in a neighborhood of regular points, i.e., points satisfying the conditions (R), $\mathscr{C}$ is a smooth submanifold of $T \mathscr{M}$. From this it follows that if $(h, \omega) \in S_{2} \times S^{2}$ is tangent to $\mathscr{C}$ at a regular point $(g, \pi)$, there is a $\delta>0$ and a smooth curve $(g(\lambda), \pi(\lambda)) \in \mathscr{C},-\delta<\lambda<\delta$, which is tangent to $(h, \omega)$ at $(g, \pi)$.

If ${ }^{(4)} h$ is a solution of (2), then ${ }^{(4)} h$ induces a solution $(h, \omega)$ of the linearized constraint equations. Thus if $(g, \pi)$ satisfies the conditions $(\mathbf{R})$, we obtain a curve of solutions to the constraint equations and by the existence theory for the Einstein equations, we get our desired curve of solutions ${ }^{(4)} g(\lambda)$ to $(1)$ which is tangent to ${ }^{(4)} h$.

3. The constraint submanifold. Let

$$
\mathscr{C}_{\mathscr{H}}=\mathscr{H}^{-1}(0)=\{(g, \pi) \mid \mathscr{H}(g, \pi)=0\},
$$


the set of solutions to the Hamiltonian constraint. Note that $k=0$ is equivalent to $\pi=0$.

THEOREM 1. Let $(g, \pi) \in \mathscr{C}_{\mathscr{H}}$ satisfy condition (ii) of (R). Then in a neighborhood of $(g, \pi), \mathscr{C}_{\mathscr{H}}$ is a smooth submanifold of $T \mathscr{M}$.

Sketch of Proof. Consider $\mathscr{H}: T \mathscr{M} \rightarrow C^{\infty}$. We show that $D \mathscr{H}(g, \pi)$ is surjective so that $\mathscr{H}$ is a submersion at $(g, \pi)$. It follows from the implicit function theorem (using Sobolev spaces here) that $\mathscr{H}^{-1}(0)$ is then a smooth submanifold in a neighborhood of $(g, \pi)$.

From A. Lichnerowicz [12] we have the classical formula

$$
D R(g) \cdot h=\Delta(\operatorname{tr} h)+\delta \delta h-h \cdot \operatorname{Ric}(g)
$$

and from this one finds

$$
\begin{aligned}
D \mathscr{H}(g, \pi) \cdot(h, \omega) & =\Delta(\operatorname{tr} h)+\delta \delta h-h \cdot \operatorname{Ric}(g) \\
& +2\left\{\frac{1}{2}(\operatorname{tr} \pi) \pi-\pi \times \pi\right\} \cdot h+2\left\{\frac{1}{2}(\operatorname{tr} \pi) g^{-1}-\pi\right\} \cdot \omega
\end{aligned}
$$

where $\pi \times \pi=\pi^{i k} \pi_{k}^{j}$. Using elliptic theory, it follows that $D \mathscr{H}(g, \pi)$ is surjective provided that its adjoint $D \mathscr{H}(g, \pi)^{*}$ is injective and has injective symbol. A straightforward computation shows that $D \mathscr{H}(g, \pi)^{*}: C^{\infty} \rightarrow$ $S_{2} \times S^{2}$ is given by

$$
\begin{array}{rl}
D & \mathscr{H}(g, \pi)^{*} \cdot N \\
=\left(g \Delta N+\operatorname{Hess} N-N \operatorname{Ric}(g)+2\left\{\frac{1}{2}(\operatorname{tr} \pi) \pi-\pi \times \pi\right\}^{b} N,\right. \\
\left.2\left\{\frac{1}{2}(\operatorname{tr} \pi) g^{-1}-\pi\right\} N\right)
\end{array}
$$

where ${ }^{b}$ denotes the covariant form of a contravariant tensor.

The symbol of $D \mathscr{H}(g, \pi)^{*}$ is

$$
\sigma_{\xi}\left(D \mathscr{H}(g, \pi)^{*}\right) s=\left(\left(-g\|\xi\|^{2}+\xi \otimes \xi\right) s, 0\right),
$$

$\xi \in T_{x}^{*} M, s \in R$, which is injective. Thus one has the $L_{2}$ orthogonal splitting $C^{\infty}=\operatorname{ker} D \mathscr{H}(g, \pi)^{*} \oplus$ range $D \mathscr{H}(g, \pi)$; see [2]. Thus if ker $D \mathscr{H}(g, \pi)^{*}=\{0\}, D \mathscr{H}(g, \pi)$ is surjective. So let $N \in \operatorname{ker} D \mathscr{H}(g, \pi)^{*}$. Then $N$ satisfies

$$
g \Delta N+\operatorname{Hess} N-N \operatorname{Ric}(g)+2\left(\frac{1}{2}(\operatorname{tr} \pi) \pi-\pi \times \pi\right)^{b} N=0
$$

and

$$
2\left(\frac{1}{2}(\operatorname{tr} \pi) g^{-1}-\pi\right) N=0 .
$$

Taking the trace of (8) gives $(\operatorname{tr} \pi) N=0$, so (8) gives $N \pi=0$. Thus, from (7),

$$
g \Delta N+\operatorname{Hess} N-N \operatorname{Ric}(g)=0
$$


whose trace gives

$$
2 \Delta N-N R(g)=0 .
$$

Using $\mathscr{H}(g, \pi)=0$ and $N \pi=0,(10)$ becomes $\Delta N=0$, so $N$ is constant. If $\pi \neq 0, N \pi=0$ implies $N=0$. If $\pi=0$, then from (7), $N \operatorname{Ric}(g)=0$. Since $(g, \pi)$ satisfies condition (ii), $g$ is not flat, so that $\operatorname{Ric}(g) \neq 0$ as we are on a 3 -manifold and so again $N=0$. Thus $D \mathscr{H}(g, \pi)^{*}$ is injective so that $D \mathscr{H}(g, \pi)$ is surjective.

If (ii) of (R) is not fulfilled, i.e., if $\pi=0$ and if $g$ is flat, then $D \mathscr{H}(g, \pi)$ is not a submersion. In fact the behavior near these points is rather different. For example, using an idea of Brill-Deser [3] one can show that if $g$ is flat, solutions of $R(g)=0$ near $g$ are also flat [10], [11].

Next we investigate the divergence constraint. Let $\mathscr{C}_{\delta} \subset T \mathscr{M}$, $\mathscr{C}_{\delta}=\left\{(g, \pi) \mid \delta \pi=-\pi_{\mid j}^{i j}=0\right\}$, the set of solutions to the divergence constraint. Using the expression $\operatorname{tr} L_{X} \pi=X \cdot d \operatorname{tr} \pi-\pi \cdot L_{X} g$, condition (iii) is easily seen to be equivalent to

(iii)' For a vector field $X, L_{X} g=0$ and $L_{X} \pi=0$ implies $X=0$.

THEOREM 3. Let $(g, \pi) \in \mathscr{C}_{\delta}$ satisfy the condition (iii)'. Then $\mathscr{C}_{\delta}$ is a smooth submanifold in a neighborhood of $(g, \pi)$.

Sketch OF Proof. One computes that $D \delta(y, \pi): S_{2} \times S^{2} \rightarrow \mathscr{X}$ is given by:

$$
D \delta(g, \pi) \cdot(h, \omega)=\delta \omega+\frac{1}{2} \pi^{j k} h_{j k}{ }^{l i}-\pi^{j k} h_{j \mid k}^{i}-\frac{1}{2} \pi^{i j}(\operatorname{tr~h})_{, j}
$$

and its adjoint $D \delta(g, \pi)^{*}: \mathscr{X} \rightarrow S_{2} \times S^{2}$ is:

$$
\begin{aligned}
D \delta(g, \pi)^{*} \cdot X=\left(\left\{-\frac{1}{2} L_{X} \pi+\right.\right. & \left.\frac{1}{2}(\delta X) \pi-\frac{1}{2}(X \otimes \delta \pi+\delta \pi \otimes X)\right\}^{\}} \\
& \left.+\frac{1}{4}\left(L_{X} g \cdot \pi\right) g-\frac{1}{2}(X \cdot \delta \pi) g, \frac{1}{2}\left(L_{X} g\right)^{-1}\right) .
\end{aligned}
$$

The symbol of $D \delta^{*}$ is again injective, so it suffices to show $D \delta(g, \pi)^{*} \cdot X=0$ implies $X=0$. Since $\delta \pi=0$, the condition $D \delta(g, \pi)^{*} \cdot X=0$ reads:

$$
\left(-\frac{1}{2} L_{X} \pi+\frac{1}{2}(\delta X) \pi\right)^{b}+\frac{1}{4}\left(L_{X} g \cdot \pi\right) g=0
$$

and

$$
L_{X} g=0 .
$$

From (14), $\delta X=0$ so (13) gives $L_{X} \pi=0$. Thus $X=0$ by (iii)'.

The regular points satisfying (iii) ${ }^{\prime}$ are just those $(g, \pi)$ having discrete isotropy group under the action of the diffeomorphism group acting on $T \mathscr{M} \approx \mathscr{M} \times S^{2}$ by pullback.

To show that $\mathscr{C}=\mathscr{C}_{\mathscr{H}} \cap \mathscr{C}_{\delta}$ is a submanifold, we need additional restrictions because there may be points at which the intersection is not transversal. At this point it is necessary to assume that $(g, \pi)$ satisfies the $\operatorname{tr} \pi=$ constant condition (which is equivalent to $\operatorname{tr} k=$ constant). 
THEOREM 4. Let $(g, \pi) \in \mathscr{C}$ satisfy conditions $(\mathrm{R})$. Then in a neighborhood of $(g, \pi), \mathscr{C}$ is a smooth submanifold.

Sketch of Proof. Consider $\Phi=(\mathscr{H}, \delta): T \mathscr{M} \rightarrow C^{\infty} \times \mathscr{X}$. We want to show $D \Phi(g, \pi)$ is surjective for $(g, \pi) \in \mathscr{C}$ and satisfying conditions (R). We know $D \Phi$ from (5) and (11). The adjoint map is given as follows:

$$
\begin{aligned}
D \Phi(g, \pi)^{*}: C^{\infty} \times \mathscr{X} & \rightarrow S_{2} \times S^{2} ;(N, X) \\
\mapsto((\Delta N) g+\operatorname{Hess} N-N \operatorname{Ric}(g) & +\left\{2\left(\frac{1}{2}(\operatorname{tr} \pi) \pi-\pi \times \pi\right) N-\frac{1}{2} L_{X} \pi\right. \\
& \left.\quad+\frac{1}{2}(\delta X) \pi-\frac{1}{2}(X \otimes \delta \pi+\delta \pi \otimes X)\right\}^{b} \\
& +\frac{1}{4}\left(L_{X} g \cdot \pi\right) g-\frac{1}{2}(X \cdot \delta \pi) g, \\
& \left.2\left\{\frac{1}{2}(\operatorname{tr} \pi) g^{-1}-\pi\right\} N+\frac{1}{2}\left(L_{X} g\right)^{-1}\right) .
\end{aligned}
$$

The symbol of this map, $\sigma_{\xi}\left(D \Phi(g, \pi)^{*}\right), \xi \in T_{x}^{*} M$, may be shown to be injective. Thus it remains to show that $D \Phi(g, \pi)^{*}$ is injective. Let $(N, X) \in \operatorname{ker}\left(D \Phi(g, \pi)^{*}\right)$. Since $\delta \pi=0,(15)$ gives

$$
\begin{aligned}
& (\Delta N) g+\operatorname{Hess} N-N \operatorname{Ric}(g) \\
& +\left\{2\left(\frac{1}{2}(\operatorname{tr} \pi) \pi-\pi \times \pi\right) N-\frac{1}{2} L_{X} \pi+\frac{1}{2}(\delta X) \pi\right\}^{b}+\frac{1}{4}\left(L_{X} g \cdot \pi\right) g=0
\end{aligned}
$$

and

$$
2\left\{\frac{1}{2}(\operatorname{tr} \pi) g^{-1}-\pi\right\} N+\frac{1}{2}\left(L_{X} g\right)^{-1}=0 .
$$

Taking the trace and using $\mathscr{H}(g, \pi)=0$ gives:

$$
2 \Delta N+2\left\{\pi \cdot \pi-\frac{1}{4}(\operatorname{tr} \pi)^{2}\right\} N-\frac{1}{2}(d \operatorname{tr} \pi) \cdot X=0
$$

and

$$
2\left\{\frac{1}{2}(\operatorname{tr} \pi) g^{-1}-\pi\right\} N+\frac{1}{2}\left(L_{X} g\right)^{-1}=0 .
$$

If $\pi=0$ then (18) gives $N=$ constant and (16) gives $N \operatorname{Ric}(g)=0$ so $N=0$, as $\operatorname{Ric}(g) \neq 0$ in this case. By (19), and (iii) of (R) we obtain $X=0$.

If $\pi \neq 0, \operatorname{tr} \pi=$ constant, so (18) gives

$$
2 \Delta N+2\left\{\pi \cdot \pi-\frac{1}{4}(\operatorname{tr} \pi)^{2}\right\} N=2 \Delta N+2\left(\pi-\frac{1}{4}(\operatorname{tr} \pi) g\right)^{2} N=0 .
$$

Since $\left(\pi-\frac{1}{4}(\operatorname{tr} \pi) g\right)^{2}=\left(\pi-\frac{1}{4}(\operatorname{tr} \pi) g\right) \cdot\left(\pi-\frac{1}{4}(\operatorname{tr} \pi) g\right)>0$, we conclude that $N=0$. Thus from (19), $L_{X} g=0$, so from (16), $L_{X} \pi=0$. Thus in either case, $X=0$, so that $D \Phi(g, \pi)^{*}$ is injective.

4. Integration of infinitesimal deformations of Ricci flat spacetimes. As explained previously, we can use Theorem 4 to prove the following main result.

THEOREM 5. Let ${ }^{(4)} g$ be a Lorentz metric on $V$ satisfying $\operatorname{Ric}\left({ }^{(4)} g\right)=0$. 
Let ${ }^{(4)} h$ satisfy the linearized equations; i.e.

$$
D \operatorname{Ric}\left({ }^{(4)} g\right) \cdot{ }^{(4)} h=\frac{1}{2}\left(\square_{L}{ }^{(4)} h-\alpha_{(4) g} \delta\left({ }^{(4)} h-\frac{1}{2}\left(\operatorname{tr}^{(4)} h\right)^{(4)} g\right)\right)=0,
$$

where $\alpha_{g}(X)=L_{X} g$ and $\square_{L}$ is the Lichnerowicz d'Alembertian [12].

Let $M$ be a compact oriented space-like hypersurface in $V$ with induced metric $g$ and second fundamental form $k$. Assume $(g, k)$ satisfy the conditions (R).

Then there exists a $\delta>0$ and a smooth curve ${ }^{(4)} g(\lambda)$ of exact solutions of $\operatorname{Ric}\left({ }^{(4)} g(\lambda)\right)=0,-\delta<\lambda<\delta$, such that ${ }^{(4)} g(0)={ }^{(4)} g$ and $d^{(4)} g(\lambda) /\left.d \lambda\right|_{\lambda=0}=$ ${ }^{(4)} h$ in a tubular neighborhood of $M$.

SKetCH OF Proof. In Gaussian coordinates in a neighborhood of $M$, ${ }^{(4)} h$ induces a deformation $(h, \omega)$ by

$$
\begin{aligned}
& \quad h_{i j}={ }^{(4)} h_{i j}, \\
& \omega=D\left(\pi^{i j}(g, \dot{g})\right) \cdot(h, \dot{h}) \quad \\
&=\frac{1}{2}\{-(h \times \dot{g}+\dot{g} \times h)+(\dot{h}-(\operatorname{tr} \dot{h}) g)+((h \cdot \dot{g}) g+(\operatorname{tr} \dot{g}) h)\}^{-1}
\end{aligned}
$$

where the momentum $\pi$ in Gaussian coordinates is

$$
\begin{gathered}
\pi=\pi^{i j}=\frac{1}{2}\left(g^{i k} g^{j l} \dot{g}_{k l}-(\operatorname{tr} \dot{g}) g^{i j}\right), \\
\dot{g}={ }^{(4)} \dot{g}_{i j}=\partial^{(4)} g_{i j} / \partial t, \dot{h}={ }^{(4)} \dot{h}_{i j}=\partial^{(4)} h_{i j} / \partial t .
\end{gathered}
$$

This induced deformation $h, \omega$ satisfies the linearized constraint equations: $D \mathscr{H}(g, \pi) \cdot(h, \omega)=0, D \delta(g, \pi) \cdot(h, \omega)=0$. Thus by Theorem 4 , we can find a curve $(g(\lambda), \pi(\lambda)) \in \mathscr{C}$ tangent to $(h, \omega)$ at $(g, \pi)$. This gives us spacetimes ${ }^{(4)} g(\lambda)$ defined on a neighborhood of $M$ by the existence theory which by a transformation of coordinates can be chosen to have the desired properties.

\section{REFERENCES}

1. R. Arnowitt, S. Deser and C. W. Misner, The dynamics of general relativity, Gravitation; an Introduction to Current Research, Wiley, New York, 1962, pp. 227-265. MR 26 \#1182.

2. M. Berger and D. Ebin, Some decompositions of the space of symmetric tensors on a Riemannian manifold, J. Differential Geometry 3 (1969), 379-392. MR 42 \#993.

3. D. Brill and S. Deser, Instability of closed spaces in general relativity (to appear).

4. - Variational methods and positive energy in relativity, Ann. Physics 50 (1968), $548-570$.

5. Y. Choquet-Bruhat and S. Deser, On the stability of flat space, (to appear); Stabilité initiale de l'espace temps de Minkowski, C.R. Acad. Sci. Paris 275 (1972), 1019-1021.

6. D. Ebin, On the space of Riemannian metrics, Proc. Sympos. Pure Math., vol. 15, Amer. Math. Soc., Providence, R.I., 1970, pp. 11-40.

7. A. Fischer, The theory of superspace, Relativity, Plenum Press, New York, 1970.

8. A. Fischer and J. Marsden, The Einstein equations of evolution-A geometric approach, J. Mathematical Phys. 13 (1972), 546-568. 
9. A. Fischer and J. Marsden, The Einstein evolution equations as a first-order quasi-linear symmetric hyperbolic system. I, Comm. Math. Phys. 28 (1972), 1-38.

10. - Submanifolds of Riemannian metrics with prescribed scalar curvature (to appear).

11. - Linearization stability of the Einstein equations (to appear).

12. A. Lichnerowicz, Propagateurs et commutateurs en relativité gènèrale, Inst. Hautes Études Sci. Publ. Math. No. 10.

13. J. A. Wolf, Spaces of constant curvature, 2nd ed., Berkeley, Calif., 1972.

Department of Mathematics, University of California, Berkeley, California 94720 